\title{
Nanoparticle diffusion within intestinal mucus: three-dimensional response analysis dissecting the impact of particle surface charge, size and heterogeneity across polyelectrolyte, pegylated and viral particles
}

Muthanna Abdulkarim ${ }^{1 .}$, Nuria Agulló ${ }^{2 .}$, Beatrice Cattoz ${ }^{3 .}$, Peter Griffith ${ }^{3 .}$, Andreas Bernkop-Schnürch ${ }^{4}$, Salvador Gómez Borros ${ }^{2}$, Mark Gumbleton ${ }^{1 .}$.

1. School of Pharmacy \& Pharmaceutical Sciences, Cardiff University, Cardiff CF14 3NB, UK

2. Grup d'Enginyeria de Materials (GEMAT), Institut Quimic de Sarrià, Universitat Ramon Llull, Via Augusta 390, 08017 Barcelona, Spain.

3. Department of Pharmaceutical, Chemical and Environmental Sciences, Faculty of Engineering and Science, University of Greenwich, Medway Campus, Central Avenue, Chatham Maritime, Kent, ME4 4TB, U.K.

4. Department of Pharmaceutical Technology, Institute of Pharmacy, University of Innsbruck, Innsbruck, Austria

Keywords: mucus, polyelectrolyte, nanoparticles, multiple particle tracking, virus, diffusion

Corresponding author :

Professor Mark Gumbleton

Redwood Building, King Edward VII Avenue

School of Pharmacy \& Pharmaceutical Sciences, Cardiff University, Cardiff CF14 3NB

Tel: $+44(0) 29-20875449$

Email: gumbleton@cardiff.ac.uk 


\section{ABSTRACT}

Multiple particle tracking (MPT) methodology was used to dissect the impact of nanoparticle surface charge and size upon particle diffusion through freshly harvested porcine jejunum mucus. The mucus was characterised rheologically and by atomic force microscopy. To vary nanoparticle surface charge we used a series of self-assembly polyelectrolyte particles composed of varying ratios of the negatively charged polyacrylic acid polymer and the positively charged chitosan polymer. This series included a neutral or near-neutral particle to correspond to highly charged but near-neutral viral particles that appear to effectively permeate mucus. In order to negate the confounding issue of self-aggregation of such neutral synthetic particles a sonication step effectively reduced particle size (to less than 340 $\mathrm{nm}$ ) for a sufficient period to conduct the tracking experiments. Across the polyelectrolyte particles a broad and meaningful relationship was observed between particle diffusion in mucus (x1000 difference between slowest and fastest particle types), particle size (104 nm to $373 \mathrm{~nm})$ and particle surface charge $(-29 \mathrm{mV}$ to $+19.5 \mathrm{mV})$, where the beneficial characteristic promoting diffusion was a neutral or near-neutral charge. The diffusion of the neutral polyelectrolyte particle $\left(0.02887 \mathrm{~cm}^{2} . \mathrm{S}^{-1} \times 10^{-9}\right)$ compared favourably with that of a highly diffusive PEGylated-PLGA particle $\left(0.03182 \mathrm{~cm}^{2} \cdot \mathrm{S}^{-1} \times 10^{-9}\right)$, despite the size of the latter (54 $\mathrm{nm}$ diameter) accommodating a reduced steric hindrance with the mucin network. Heterogeneity of particle diffusion within a given particle type revealed the most diffusive $10 \%$ sub-population for the neutral polyelectrolyte formulation $\left(5.809 \mathrm{~cm}^{2} \cdot \mathrm{S}^{-1} \times 10^{-9}\right)$ be faster than that of the most diffusive $10 \%$ sub-populations obtained for either the PEGylatedPLGA particle $\left(4.061 \mathrm{~cm}^{2} . \mathrm{S}^{-1} \times 10^{-9}\right)$ or for a capsid adenovirus particle $\left(1.922 \mathrm{~cm}^{2} . \mathrm{S}^{-1} \times 10^{-}\right.$ $\left.{ }^{9}\right)$. While this study has used a simple self-assembly polyelectrolyte system it has substantiated the pursuance of other polymer synthesis approaches (such as living free-radical polymerisation) to deliver stable, size-controlled nanoparticles possessing a uniform high density charge distribution yielding net neutral surface potential. Such particles that will provide an additional strategy to that of PEGylated systems where the interactions of mucosally-delivered nanoparticles with the mucus barrier are to be minimised. 


\section{INTRODUCTION}

Safeguarding the stability and effective delivery of biopharmaceuticals within the gastrointestinal (GIT) lumen is addressed in a number of ways the most usual of which is the formulation of the therapeutic into a protective nanoparticle (NP). Such NPs which may release cargo in close proximity to the absorption barrier or, as a fully intact particle, be transported across the epithelium [1]. Irrespective of the overall strategy the permeation of NPs through the extracellular covering of mucus to reach the underlying epithelial surface is recognised as a significant limitation [2].

The mucus barrier comprises a firmly adherent unstirred viscous layer directly adjacent to the epithelium, and a loosely adherent less viscous layer more distal to the epithelium. The main constituents of mucus are: mucin glycoproteins, lipids, and sloughed cellular material. It is the high molecular weight mucin glycoprotein that is the main component restricting the movement of particles. Specifically, particle permeation through the mucus gel is recognised to be affected by several types of interactions, including: contact of the particle with the hydrophobic domains of the mucin polymer; steric hindrance physically restricting particle movement through the porous water-filled channels of the mucus gel network [3]; cohesive and repulsive electrostatic contacts of the particle with, in particular, the dense coat of glycans covering the mucin fibre which display charged sulphate or carboxylate groups $[4,5]$.

The concept of modifying a particle's surface characteristics to enhance permeation through mucus was first described by Lai et al [6], and has most commonly involved the generation of a polar particle surface by various PEGylation strategies. For example, Hanes' group [7] has shown that while particle size per se has some role in determining mucus permeation it is the particle's surface characteristics that can have a more profound impact, e.g. PEGylation of a $500 \mathrm{~nm}$ polystyrene particle increasing permeation by $>1000$-fold. In contrast to synthetic particles, some viral particles are well adapted for efficient mucus gel permeation, for example, the Norwalk virus $(38 \mathrm{~nm})$ and human Papilloma virus (55nm) appear to penetrate mucus in an unrestricted manner [8]. The general features enabling the permeation of such viruses appear to be their small size, the lack of a hydrophobic envelope, and the possession of an exposed hydrophilic protein capsid shell characterised by a high surface density of +/- charges giving a net membrane potential close to neutrality [8,9]. However, the ability to mimic the surface characteristics of a virus is a challenge when constructing synthetic particles not least, because of the tendency toward particle aggregation as surface charge approaches neutrality. Nevertheless, there is some evidence that a synthetic particle 
of near-neutral surface charge $(+0.9 \mathrm{mV})$, and comprising polyacrylic acid (PAA; -ve) and polyallylamine (PAM; +ve) polymers, does show improved mucus permeation, at least in an in-vitro side-by-side diffusion chamber model [10]. The approach of generating a polar, electrically neutral surface on a synthetic particle has been described as mediating a particle's 'slip' through mucus [11].

Using multiple particle tracking (MPT) methodology this current work explored the impact upon NP diffusional kinetics through mucus of the variables of particle surface charge and size. Polyelectrolyte NPs of various surface charge were formulated by combining different ratios of PAA (-ve) and chitosan (+ve) polymers. The study used freshly harvested and characterised (rheologically and by surface morphology) porcine jejunum intestinal mucus. In particular we sought to examine the diffusion of a synthetic particle possessing a charged but electrically neutral surface. To address the tendency of neutral polyelectrolyte particles to self-aggregate we adopted a sonication step that effectively reduced the size of large neutral particles for a sufficient period to conduct MPT. To account for the impact of a particle's size upon its unrestricted diffusion in solution we referenced the particle diffusion data in mucus to the respective data in water. Beyond diffusion coefficients per se we report on the proportion of diffusive particles in each formulation, and the heterogeneity of diffusion within sub-populations of a given particle type. Comparison was also made to a non-charged hydrophilic particle, PLGA-PEG, and to a capsid virus with a near neutral surface charge. Even for the simple polyelectrolyte systems we found neutral or near-neutral charged particles to display a significant advantage in terms of diffusion in mucus. This finding substantiates the pursuance of other polymer synthesis approaches ultimately yielding stable particles with a high charge density but net neutral surface potential that will provide minimal particle interactions with the mucus barrier.

\section{MATERIALS AND METHODS}

\section{Materials}

The chitosan polymer (low MW), polyacrylic acid (PAA) polymer (MW 1800) and MES buffer were obtained from Sigma-Aldrich (UK). PLGA (PDLG-5002 containing lactic:glycolic at 50\%:50\%, MW 17 KDa.) and PEGylated-PLGA (mPEG MW 5 KDa. at 85\%PLGA: 15\% mPEG) and Lumogen Red-305 (LR) (excitation $573 \mathrm{~nm}$ emission $612 \mathrm{~nm}$ ) were all supplied by Nanomi (Netherlands). The amplified AD5 virus was kindly provided by 
the Institute of Cancer Genetics (Cardiff University). Alexa Fluor® 488 Protein labelling kit was from Life Technologies (UK). Glass bottom imaging dishes (35 $\mathrm{mm}$ diameter dish with a glass coverslip at $1.5 \mathrm{~mm}$ thick and $10 \mathrm{~mm}$ diameter) were from MatTek Corporation (USA). All other reagents and solvents were from Fisher Scientific (Loughborough, UK).

\section{Mucus}

Freshly isolated pig intestinal ileum ( $2 \mathrm{~m}$ in length from proximal region) was obtained from a local abattoir (Cardiff) and kept in ice-cold oxygenated phosphate buffered saline (PBS) (no longer than $2 \mathrm{hrs}$ ) prior to sample processing. The ileum was processed into $25 \mathrm{~cm}$ lengths with each length incised longitudinally to allow intestinal food and other waste debris to be was gently rinsed away by ice-cold PBS. The mucus was then harvested by an approach [2] recognised to optimise the yield of not only the loose mucus layer but critically also a high content of the adherent mucus layer. Simply it involved gentle scraping from the intestinal surface by spatula avoiding the shedding of significant intestinal epithelial tissue. Mucus was divided into aliquots $(0.5 \mathrm{~g})$ and kept at $-20{ }^{\circ} \mathrm{C}$ prior to experimentation [12]

\section{Methods}

\section{Nanoparticle preparation}

Polyelectrolyte NPs were prepared from chitosan and polyacrylic acid (PAA) polymers which respectively served as the source for the positive and negative charges. Stock solutions $(1 \% \mathrm{w} / \mathrm{v})$ of chitosan and PAA were prepared in $1 \%$ glacial acetic acid and in distilled deionised (DD) water, respectively. Working solutions for both polymers were prepared by diluting the stock solutions to $0.02 \% \mathrm{w} / \mathrm{v}$ with MES buffer $\mathrm{pH} 6.5$. The polyelectrolyte NPs were synthesized by the self-assembly technique [13] whereby the dilute solution of chitosan was added drop wise (1 drop/minute) to the dilute solution of PAA and the mixture stirred magnetically for $16 \mathrm{hrs}$. The use of MES buffer in the NP self-assembly process avoided precipitation issues evident when phosphate or citrate buffers were used.

Lumogen Red-305 (LR) was loaded into the NPs by the coacervation technique [14], whereby LR dissolved $(0.2 \% \mathrm{w} / \mathrm{v})$ in acetone was added to the dilute solution of PAA prior to the addition of chitosan. The mass of LR added equated to $150 \mu \mathrm{g}$ LR per $30 \mathrm{mg}$ of combined PAA:chitosan polymer. The acetone was evaporated during the mixing allowing LR loading to the core of the polyelectrolyte NPs. All particles were subject to a $2 \mathrm{hr}$ stability test for size and zeta-potential (see below) prior to capture of multiple particle tracking data. 


\section{Nanoparticle sonication}

Polyelectrolyte NPs displaying a neutral surface charge (i.e. PAA:chitosan mass ratios of 1: 2.2) gave rise to large particles. Given a key aim of the study to investigate the relative significance of charge and size as independent variables, these large neutral particles were temporarily reduced in size by probe sonication. Accordingly, suspensions of the neutral NPs (ca $1200 \mathrm{~nm}$ ) were sonicated (Fisher scientific probe sonicator, UK) for $2 \mathrm{~min}$. at $20 \mathrm{KHz}$ and $70 \%$ amplitude with the suspension then syringe-filtered through $0.45 \mu$ membrane (Millipore, UK) Particle size and zeta potential were measured immediately after filtration and over a subsequent test period ( $2 \mathrm{hr}$ ) to ensure a sufficient duration of size stability to enable the conduct of the mucus diffusion studies. To serve as controls for the sonication process both positively-charged (i.e. PAA:chitosan mass ratio of $1: 8$ ) and negativelycharged (i.e. PAA:chitosan mass ratio of $1: 1$ ) polyelectrolyte NPs were also subject to the sonication process and their diffusion characteristics (before and after) were determined.

\section{Zeta potential measurement}

A Malvern NANO ZS (Malvern, UK) was used to measure the zeta potential of the NP samples. The instrument was standardised prior to each experiment by use of calibration standards.

\section{Particle size measurement}

Particle size (diameter) was measured by photon correlation spectroscopy (Malvern NANO ZS) with data collected in uni-modal setting. The instrument was standardised prior to each experiment by use of calibration standards.

\section{Rheological studies on mucus}

The measurement of the elastic modulus (Storage) and the viscous modulus (Loss) was undertaken using an IR550 rheometer (TA Instruments, USA) (University Ramon Llull, Barcelona, Spain). The strain sweep test was conducted using $40 \mathrm{~mm}$ diameter parallel plates with a gap width of $1 \mathrm{~mm}$. An oscillating amplitude mode was applied at up to 95\% strain in which the plateau stress curve is obtained and the Loss $\left(\mathrm{G}^{\prime \prime}\right)$ and Storage $\left(\mathrm{G}^{\prime}\right)$ modulus recorded in Pascal. The phase angle $(\delta)$ was calculated by the equation: $\tan \delta=\left(\mathrm{G}^{\prime \prime} / \mathrm{G}^{\prime}\right)[15]$.

\section{Atomic Force Microscopy (AFM) imaging of mucus}


AFM imaging (XE-100 Advanced Scanning Probe Microscope, Park Systems, Korea) was used to assess the surface morphology of the pores in the mucus network. Samples were scanned at a large format of $25 \times 25 \mu \mathrm{m}$ then zoomed to $15 \times 15 \mu \mathrm{m}$ and $5 \times 5 \mu \mathrm{m}$ formats. For each format 10 images were acquired at random locations. From each image the diameters of all visible pores were measured and the overall distribution of pore diameters assessed.

\section{Multiple particle tracking (MPT) in mucus}

NP diffusion through intestinal mucus was assessed by MPT technique using previously described mathematical approaches $[6,7,16,17]$. Samples $(0.5 \mathrm{~g})$ of porcine intestinal mucus were incubated in glass-bottom MatTek imaging dishes at $37{ }^{\circ} \mathrm{C}$. The fluorescently labelled NPs were inoculated into each $0.5 \mathrm{~g}$ mucus sample in a $25 \mu \mathrm{l}$ aliquot at a suspension concentration of $0.002 \%$ NPs, a dilution confirmed to reproducibly avoid particle aggregation at the point of inoculation into the mucus sample. To ensure effective particle distribution following inoculation within the mucus a $2 \mathrm{hr}$ period of equilibration was adopted prior to beginning video microscopy capture of NP movements. Video capture involved 2dimensional imaging on a Leica DM IRB wide-field epifluorescence microscope (x63 magnification oil immersion lens) using a high speed camera (Allied Vision Technologies, $\mathrm{UK}$ ) running at a frame rate of $33 \mathrm{~ms}$ i.e. capturing 30 frames $\mathrm{sec}^{-1}$; each completed video film comprised 300 frames. For each 0.5 g mucus sample approximately 120 NPs were simultaneously tracked and their movements captured. For any distinct NP species, e.g. a particular polyelectrolyte mass ratio, a minimum of three distinct mucus samples were analysed, i.e. minimum of 360 individual NP trajectories assessed.

The tracking (over a maximum of 10 seconds) of fully intact discrete particles was defined by microscopic criteria, and particle size and zeta potential stability over $2 \mathrm{hr}$. (see above); any fluorescent dye dissociated from the surface of the particle contributed only to the background fluorescence and not to the appearance of distinct microscopic particles. Supplementary videos 1, 2 and 3 show respectively the video capture of the $334 \mathrm{~nm}$ diameter polyelectrolyte neutral particles, i.e. [F8] formulation, the $54 \mathrm{~nm}$ PEG-PLGA particles and the $146 \mathrm{~nm}$ AD5 capsid virus.

Videos were imported into Fiji ImageJ software to convert the movement of each NP into individual NP trajectories across the full duration of the $10 \mathrm{sec}$. videos. However, for the analysis of particle diffusion only a 30 frame video period (1 sec) was used, with the criterion that any individual particle tracked must display a continuous presence in the $\mathrm{X}-\mathrm{Y}$ plane 
throughout the respective 30 sequential frames. Limiting the period of analysis to 30 frames minimised the impact of mucin movement upon the particle diffusion calculations [6]. The individual particle trajectories were converted into numeric pixel data (Mosaic Particle Tracker within Fiji ImageJ) which, based on the microscope and video capture settings, was converted into metric distance. The distances moved by every individual particle over a selected time interval $(\Delta \mathrm{t})$ in the $\mathrm{X}-\mathrm{Y}$ trajectory were then expressed as a squared displacement (SD). The mean square displacement (MSD) of any single particle (n) represents the geometric mean of that particle's squared displacements throughout its entire 30 -frame trajectory.

MSD was determined as follows [18]:

$\operatorname{MSD}_{(\mathrm{n})}=\left(\mathrm{X}_{\Delta \mathrm{t}}\right)^{2}+\left(\mathrm{Y}_{\Delta \mathrm{t}}\right)^{2} \quad$ Equation 1

In any single mucus sample experiment an MSD was calculated for at least each of 120 individual particles (i.e. 120 MSD calculations) with the experiment replicated a further two times for any particle type, i.e. at least 360 particles studied in total. For each NP type under study (e.g. PEG-PLGA) an "ensemble mean square displacement" (defined by $\langle$ MSD>) was then determined for each of the three replicate studies. The Effective Diffusion Coefficient $(<$ Deff $>$ ) for a particular NP type was then calculated by:

$\langle$ Deff $\rangle=\langle\operatorname{MSD}\rangle /(4 * \Delta \mathrm{t}) \quad$ Equation 2

Where 4 is a constant relating to the 2-dimensional mode of video capture and $\Delta t$ is the selected time interval.

Proportion of diffusive particles: Measuring particle diffusion across various time intervals allows for a description of the proportion of particles that are diffusive through the mucus matrix [6].

Equation 3 was used to determine a Diffusivity Factor (DF) which expresses the effective diffusion coefficient for each individual particle (Deff) across the time intervals $(\Delta t)$ of 1 sec. and $0.2 \mathrm{sec}$.

$\mathrm{DF}=\operatorname{Deff}_{\Delta \mathrm{t}=1 \mathrm{sec}} /$ Deff $_{\Delta \mathrm{t}=0.2 \mathrm{sec}} \quad$ Equation 3 
Where the individual particle Deff $=\mathrm{MSD} /(4 * \Delta \mathrm{t})$. Particles with a DF value of 0.9 and greater were defined as diffusive. The proportion of the diffusive particles within a given NP type under study was then calculated and expressed as \%Diffusive particles.

Heterogeneity in particle diffusion: Profiling the diffusive properties of each particle within an entire population provides information on the heterogeneity of particle movement and the presence of outlier sub-populations that may follow distinct pathways of diffusion through the matrix. Here the effective diffusion coefficient for each individual particle (Deff) was calculated at the time interval $(\Delta t)$ of $1 \mathrm{sec}$, and for any NP type all $360 \operatorname{Deff}_{\Delta t=1} \mathrm{sec}$ were then ranked to allow comparison of the highest $\left(90^{\text {th }}\right)$ and lowest $\left(10^{\text {th }}\right)$ percentiles, where for example the $90^{\text {th }}$ percentile is the Deff value below which $90 \%$ of the Deff observations may be found.

\section{Particle diffusion in water}

The particles' diffusion coefficient $\left(\mathrm{D}^{\circ}\right)$ in water was calculated by the Stokes-Einstein equation at a temperature of $37 \mathrm{C}^{\circ}[19]$ :

$\left[\mathrm{D}^{\circ}=\kappa \mathrm{T} / 6 \pi \eta \mathrm{r}\right] \quad$ Equation 4

Where $\kappa$ is Boltzmann constant, $\mathrm{T}$ is absolute temperature, $\eta$ is water viscosity and $\mathrm{r}$ is radius of the particle.

The diffusion of all particles were also expressed as the parameter, \%ratio [Deff] $/\left[\mathrm{D}^{\circ}\right]$.

\section{Statistical analysis}

One-way ANOVA and Bonferonni multiple comparison tests were used for the comparative analysis between multiple particle types. A significance level $\mathrm{p}<0.05$ was applied.

\section{RESULTS AND DISCUSSION}

Intestinal mucus represents a primary barrier for the access of NP to the enterocyte surface where release of therapeutic cargo is ideally triggered. In this work we used MPT methodology to explore the NP diffusivity through porcine intestinal mucus and how diffusivity was influenced by the variables of particle surface charge and size. To explore the impact of these variables we chose to use a series polyelectrolyte NP formulations composed of variable ratios of the charged polymers PAA (-ve) and chitosan (+ve). The model provided a simple two component chemistry self-assembly system providing particles of 
variable surface charge. In particular we sought to determine the comparative effectiveness of highly charged but neutral or near-neutral particles to diffuse through mucus. Our enquiry was underpinned by the effectiveness of some viral particles to permeate through mucus not only as a result of their small particle dimensions, but also through possession of a highly charged near-neutral exterior facing surface. Our multi-variate analysis of polyelectrolyte particle diffusion also included comparison to the diffusion kinetics of a capsid virus with a near neutral surface charge, and to a non-charged hydrophilic particle, i.e. PLGA-PEG, the nature of which has previously been shown to facilitate high diffusivity in cervico-vaginal mucus [6]. Even for the simple polyelectrolyte systems we found neutral or near-neutral charged particles to display a significant advantage in terms of diffusion. This finding substantiates the pursuance of other polymer synthesis approaches, such as living free radical chemistry, to deliver stable, size-controlled nanoparticles possessing a uniform high density distribution of positive and negative surface charges to ultimately yield a net neutral surface potential. Such particles that will provide an additional strategy to that of PEGylated systems when interactions with the mucus barrier are to be minimised.

Table 1 shows the zeta potential and particle size measurements of the polyelectrolyte NPs formulated from the PAA and chitosan polymers. The Effective Diffusion Coefficient $(<$ Deff $>)$ in mucus is shown for each NP type, also shown is the respective Diffusion Coefficient in water $\left(\mathrm{D}^{\circ}\right)$. Moreover, the ratio (expressed as \%) of these two parameters, $(\%$ ratio $\left\langle\right.$ Deff $>/ \mathrm{D}^{\circ}$ ) provides a measure of the relative efficiency of particle diffusion through mucus when the particles' intrinsic free Brownian motion in water is taken into account. As such it affords comparison of particle diffusion in mucus after accounting for the impact of a particle's size upon its unrestricted diffusion in solution. It is essentially a measure that more directly addresses the relative impact between particles of differing surface physico-chemical properties and the interactions with, and the steric hindrance of, the mucin network.

Rheological assessment of the mucus showed it to possess a gel structure defined by a storage modulus $\left(\mathrm{G}^{\prime}\right)$ that was greater than the loss modulus $\left(\mathrm{G}^{\prime \prime}\right)$ (Supplementary Figure 1.) and a $\delta$ of less than $45^{\circ}$ [20]; specifically $\delta$ values were recorded between 14.82 to $16.22^{\circ}$. Supplementary Figure 2a shows the surface morphology of the mucus with large $(25 \times 25 \mu \mathrm{m})$, medium $(15 \times 15 \mu \mathrm{m})$ and small $(5 \times 5 \mu \mathrm{m})$ field AFM images of the pore characteristics of the mucus network. Supplementary Figure $2 b$ shows a histogram of the size distribution of over 98\% of all AFM visualised pores within the mucus gel across ten randomly selected AFM fields each of $25 \times 25 \mu \mathrm{m}$. The mucus harvesting approach that was used is one recognised to 
contain both loose and adherent mucus layers [2,21], and consistent with this we found the pore population to be comparatively heterogeneous with an approximate 7-fold range in the pore size from $60 \mathrm{~nm}$ to $400 \mathrm{~nm}$, but with a distribution defined by approximately $50 \%$ of the pores below $200 \mathrm{~nm}$ in diameter and $33 \%$ between 300 to $400 \mathrm{~nm}$.

The polyelectrolyte NPs possessed a surface charge ranging from $-29 \mathrm{mV}$ to $+19 \mathrm{mV}$ and a hydrodynamic diameter ranging between $104 \mathrm{~nm}$ to $373 \mathrm{~nm}$ (Table 1). The exception to this was the neutrally charged $(-0.5 \mathrm{mV})$ polyelectrolyte particle [F7] which, lacking repulsive electrostatic or steric hindrance forces, aggregated to a size of $1244 \mathrm{~nm}$. While more generally there was no obvious pattern between polyelectrolyte particle diameter and <Deff> (Figure 1a), it is clear that size had a significant role in restricting the movement of [F7] compared to its smaller derivative particle [F8] $(+1.1 \mathrm{mV}, 334 \mathrm{~nm})$, the latter displaying an approximate 12-fold greater <Deff $>$ (Figure 1b). The [F8] particle was derived by sonication and filtration of [F7] specifically with the aim to provide a neutral particle whose size was consistent with the other polyelectrolyte NP formulations. The size stability of [F8] in water was confirmed over a time period of $2 \mathrm{hr}$, sufficient to allow its physical characterisation and the conduct of the MPT experiments; the absence of re-aggregation of these particles in mucus during the MPT experiment itself was confirmed by fluorescence microscopy. To address if the sonication process may have led to an increase in particle diffusion by mechanisms beyond a reduction in particle size per se, the largest +ve particle, [F5], and largest -ve particle, [F13], were also subjected to the sonication process; neither of the respective derived particles, [F6] and [F14], showed enhanced diffusion. The more general issue of size-dependency in particle diffusion through mucus appears to be more discernible for particles of a consistent surface chemistry. For example, Dawson et al. [22] using either amine or carboxylate terminated polystyrene NPs showed size-dependency (range $100 \mathrm{~nm}$ to $500 \mathrm{~nm}$ ) in particle diffusion within cystic fibrosis sputum. Similarly, the movement of DNA through cervical mucus has been shown to be dependent upon DNA particle diameter in the range 115 to $310 \mathrm{~nm}$ [23]. Nevertheless, the effect of particle size upon diffusion within mucus is just one variable and, depending upon context, the surface properties can potentially be more significant.

The polyelectrolyte particles in the current work displayed zeta-potentials ranging between $30 \mathrm{mV}$ [F1] to $+20 \mathrm{mV}$ [F14] (Table 1). An essentially neutral zeta potential was obtained at a PAA:chitosan mass ratio of 1:2.2 [F7, F8], a mass ratio consistent with the PAA polymer possessing a relatively higher density of negatively charged groups compared to the density 
of positive charges on each chitosan molecule [24]. Particles less than $365 \mathrm{~nm}$ and which displayed the greater <Deff> (Figure 1c, Table 1) were those which bore negative $(-25 \mathrm{mV}$ and greater) (i.e. particles [F3-F6]) or near neutral surface charge (i.e. [F8] and [F9]). For example, the [F5] particle $(-15.4 \mathrm{mV})$ showed an approximate 14-fold greater $\langle$ Deff $>$ than the $[\mathrm{F} 1]$ particle $(-29.1 \mathrm{mV})$ despite the former being of a larger size $(357 \mathrm{~nm}$ vs $104 \mathrm{~nm})$. Positively charged particles of greater than $+14 \mathrm{mV}$ displayed a $<$ Deff $>$ that was at least $x 10-$ fold lower than the neutral [F8] formulation. Generally the positively charged particles displayed a much reduced diffusion compared to the negatively charged species. For the positive particles the increasing content of chitosan provides greater opportunity for the NPs to interact through electrostatic forces with mucin. However, chitosan interactions with mucin are complex, and while electrostatic attraction may be the major mechanism of interaction at high chitosan content, the impact of progressive charge-shielding of chitosan's primary amino groups by PAA will reveal significant hydrophobic and H-bonding attractive forces For the positive particles the increasing content of chitosan provides greater opportunity for mucin to interact through both electrostatic and hydrophobic forces with the chitosan polymer $[25,26]$. The carboxylate groups of the PAA polymer will itself provide opportunity for H-bonding interactions with mucin [27].

Figure 2. uses the data from Table 1 to show a 3-dimensional response analysis of polyelectrolyte NP diffusion <Deff> through mucus as a function of both particle size and zeta potential. The crucial impact of zeta-potential is clear with a 'backbone of greatest diffusion' corresponding with particles of neutral surface charge. Figure 1E shows the relative permeation of the polyelectrolyte particles in mucus expressed as \%ratio [Deff] $\left[\mathrm{D}^{\circ}\right]$ and plotted against zeta-potential. Here, after accounting for the impact of a particle's size upon its unrestricted diffusion in solution, the superior performance of the neutral ([F8], +1 $\mathrm{mV})$ is more profound, with this particle displaying a significantly greater $(\mathrm{P}<0.05)$ diffusion ratio than any other polyelectrolyte formulation.

By determining the Effective Diffusion Coefficient for each individual particle (Deff) across the time intervals of $1 \mathrm{sec}$. and $0.2 \mathrm{sec}$. we were able to calculate the proportion of the entire population of particles that can be defined as diffusive through the mucus [6]. Supplementary Figure 3. illustrates individual Deff measurements for 20 randomly selected particles (from the neutrally charged sonicated [F8] polyelectrolyte formulation) across the time intervals of $0.2 \mathrm{sec}$. and $1 \mathrm{sec}$. The vast majority of these measurements show a decline in Deff as the monitoring interval is extended to $1 \mathrm{sec}$. compared to the $0.2 \mathrm{sec}$. interval. This is interpreted 
as the occurrence with time of increased interactions of a particle with the micro-domains of the mucin network. Some particles, however, display a rise in Deff across the $1 \mathrm{sec}$. interval indicative of free diffusive transport, and it is those particles that display such an increase that are taken to represent the proportion of diffusive particles for any particle type, i.e. for [F8] the proportion of diffusive particles was $13 \%$ (Table 1) and was significantly greater $(\mathrm{P}<0.05)$ than for all other polyelectrolyte particles despite its greater size of $334 \mathrm{~nm}$. Examining particle diffusion over these short time scales substantially eliminates the effect that the contractile and stretching behaviour of the mucin fibres themselves may have on particle movement [28]. It is noteworthy that the difference in $\langle$ Deff $\rangle$ between the neutrally charged [F7] and [F8] particles (i.e. an approximate x12-fold difference) was directly proportional to their respective proportion of Diffusive particles, i.e. $1 \%$ to $13 \%$, respectively.

The PEGylation of particles is aimed at providing a uniform uncharged hydrophilic external layer that minimises hydrophobic and electrostatic interactions of the particle's surface with, for example, the hydrophobic domains or glycosidic linkages of the mucin fibres [29]. Indeed PEGylated NPs have been shown to display high permeation through cervical-vaginal mucus [30] and within sputum from cystic fibrosis patients [31]. Not surprisingly on most measures of particle diffusion through mucus we found the PEGylated-PLGA particle was superior to any other particle examined. For example, the $<$ Deff $>$ of the PEG-PLGA particle was approximately x15-fold greater than that of the neutral [F8] particle (Table 1, Figures 1B, 1D). Similarly, the proportion of diffusive particles in the PEGylated-PLGA formulation was substantially greater $(\mathrm{P}<0.05)$ at $32 \%$ than for any other particle type examined (Table 1$)$. Nevertheless, when accounting for the impact of particle size and unrestricted particle diffusion in solution, then the advantage of PEG-PLGA particle over the neutral [F8] polyelectrolyte particle is less pronounced, i.e. the \%ratio $[$ Deff $] /\left[\mathrm{D}^{\circ}\right]$ was only x 2.6 -fold greater (Figure 1F, Table 1), despite the size of the PEG-PLGA particle $(54 \mathrm{~nm})$ accommodating a reduced steric hindrance with the mucin network (Figure 1F, Table 1).

Some capsid virus particles can display rapid permeation through a mucus gel, e.g. Norwalk virus and human papilloma virus [8]. Of importance in this context is the lack of significant steric effects facilitated by the virus' relatively small geometry (typically <100 nm). However, as for synthetic particles the electrostatic stability of the virus particle in extracellular conditions is critical [32] and capsid viruses that permeate mucus effectively tend to possess an external facing surface that comprises a high density of positive and negative charges while presenting an essentially electrically neutral or near-neutral character, 
i.e. properties that minimising hydrophobic and electrostatic interactions. For example, Bozic et al [33] analysed the spatial distribution of positive and negative charges across the capsid wall of 130 viral capsids and reported the majority to possess an external charge close to zero, or slightly negative. Consistent with this Michen and Graule [34] evaluated the isoelectric points (IEP) of a number of viruses species, some of which are known to infect through intestinal or pulmonary mucus. They reported, for example, the IEP for the Norwalk virus to be 5.5 - 6.0, for the Polio virus to be $6.6-7.4$, for the Rotavirus A to approximate 8.0, and the Influenza A virus to be $6.5-7.0$.

In this study we tracked the diffusion of the neutrally charged $(-0.5 \mathrm{mV})$ adenovirus AD5 particle (size $145 \mathrm{~nm}$ ) through intestinal mucus. The determined <Deff> of the AD5 particle indeed ranked highly, second only to the much smaller PEG-PLGA particle (54 nm) but only some 1.5-fold greater than the much larger sized neutral polyelectrolyte [F8] formulation (334 nm) (Figures 1B and 1D, Table 1). However, when accounting for the impact of unrestricted particle diffusion in solution, then the neutral polyelectrolyte [F8] particle now showed a slightly improved (x1.5 fold greater) mucus permeation over the AD5 particle despite the greater size and likelihood of steric hindrance in the mucus (Figure 1F, Table 1).Clearly, while the AD5 particle displayed a neutral surface charge other determinants such as interaction of the capsid proteins will have likely impacted, at least in this study, on mucus permeation. Indeed glycocalyx and tethered mucin within the respiratory tract have been shown to inhibit adsorption of certain adenovirus serotypes to lung tissue $[35,36]$.

The heterogeneity in the movement of individual particles through mucus can provide an insight into how different subpopulations within a given formulation (particle) type may exploit divergent permeation pathways. Here we assessed such heterogeneity by measuring over a time interval of $1 \mathrm{sec}$. the effective diffusion coefficient (Deff) for each of 360 individual particles from any given particle type, and ranking this Deff data into percentiles. Figure 3 shows the percentile data for four different particle types. Generally, the more positively or negatively charged the particle type then the less heterogeneous was the diffusion data. For example, for the highly negative (104 nm sized) [F1] formulation some $90 \%$ of the particles displayed a Deff $\left(\mathrm{cm}^{2} . \mathrm{S}^{-1} \times 10^{-9}\right)$ below 0.024 , while $10 \%$ of the particles had a Deff below 0.00015 (Figure $3 \mathrm{a}$ ). The resulting ratio $90^{\text {th }} / 10^{\text {th }}$ percentile was 150 . For the highly positive (373 nm sized) [F14] particle the corresponding ratio was 180 (Figure 3b). It is not unreasonable that the more highly charged polyelectrolyte particles will display a more homogenous physical character (size, charge), and as such the variability in forces 
influencing particle diffusion through the mucus will be determined predominantly by the structure of the mucus mesh itself rather than any distinct particle subpopulations [22]. Similarly, the PEG-PLGA particle (54 nm sized) exhibited a $90^{\text {th }} / 10^{\text {th }}$ percentile ratio of 200 (Figure 3d), consistent with previously published PEGylated particle diffusion through mucus [6]. In contrast, the sonicated (334 $\mathrm{nm}$ sized) neutrally charged [F8] particle displayed a marked heterogeneity with a $90^{\text {th }} / 10^{\text {th }}$ percentile ratio of 11,000 (Figure $3 \mathrm{c}$ ). This is not readily attributable solely to the heterogeneous structure of the mucus mesh but must reflect additional diversity involving particle subpopulations and differential forces hindering their movement. Creating a charged but neutral synthetic particle through the simple process of self-assembly of polyelectrolyte polymers will inevitably lead to opposing surface charges that are not uniformly distributed with the result of forming surface 'hotspots' or surface domains of a particular electrical sign that will have differential interactions with the mucin network. Indeed, the spatial arrangement of charge on a particle's surface, even for particles of the same overall net surface charge, has been shown to be influential in particle transport through a mucin gel [37]. Such heterogeneity reflects one of the limitations of creating a charged but neutral synthetic particle through the simple process of self-assembly of polyelectrolyte polymers. Nevertheless, the fastest particles $\left(90^{\text {th }}\right.$ percentile) seen for the neutral polyelectrolyte [F8] formulation (i.e. Deff of 5.81; Figure 3c) were at least as fast as the $90^{\text {th }}$ percentile particles recorded for the much smaller PEG-PLGA (i.e. Deff of 4.06; Figure 3d).

In summary, viral particles that possess a highly charged but near-neutral exterior facing surface permeate effectively through mucus. As such we sought in this study to determine how such properties conveyed to synthetic particles would impact upon their comparative diffusion within a mucus matrix. Using multiple particle tracking methodology we explored the impact of surface charge and size upon the diffusion of synthetic particles through freshly harvested porcine jejunum mucus. The principle nanoparticle model was a simple selfassembly polyelectrolyte system comprising variable ratios of the negatively charged PAA polymer and the positively charged chitosan polymer. To address the tendency of neutral polyelectrolyte particles to self-aggregate we adopted a sonication step that effectively reduced the size (to less than $340 \mathrm{~nm}$ ) of the polyelectrolyte neutral particles for a sufficient period to conduct the tracking experiments. Further, by referencing the mucus particle diffusion data in mucus to that in water we accounted for the impact of a particle's size upon its unrestricted diffusion in solution. The results revealed a broad and meaningful 
relationship between particle diffusion in mucus (x1000 difference between slowest and fastest particle types), particle size $(104 \mathrm{~nm}$ to $373 \mathrm{~nm})$ and particle surface charge $(-29 \mathrm{mV}$ to $+19.5 \mathrm{mV}$ ). In particular the crucial impact of zeta-potential was clear, with highest diffusion in mucus (from amongst the series of polyelectrolyte particles) observed for those of a neutral surface charge (e.g. particle [F8] at $+1.1 \mathrm{mV}, 334 \mathrm{~nm}$ ). The diffusion of the neutral polyelectrolyte particle compared favourably with that of a highly diffusive PEGylated-PLGA particle $(54 \mathrm{~nm})$ despite the size of the latter accommodating a reduced steric hindrance with the mucin network. Assessment of the heterogeneity of diffusion within a given particle type revealed the most diffusive neutral polyelectrolyte nanoparticle formulation ([F8]) to display a x11,000-fold difference in particle diffusion between the fastest $10 \%$ sub-population and the slowest $10 \%$ sub-population; a finding likely reflecting a non-uniform distribution of surface charge expected for a neutral particle of this type. Of significance the most diffusive $10 \%$ sub-population for the neutral polyelectrolyte formulation $\left(5.809 \mathrm{~cm}^{2} \cdot \mathrm{S}^{-1} \times 10^{-9}\right)$ was faster than the most diffusive $10 \%$ sub-populations obtained for either the PEGylated-PLGA particle $\left(4.061 \mathrm{~cm}^{2} \cdot \mathrm{S}^{-1} \times 10^{-9}\right)$ or for a capsid adenovirus particle $\left(1.922 \mathrm{~cm}^{2} \cdot \mathrm{S}^{-1} \times 10^{-9}\right)$. While this study has used a simple self-assembly polyelectrolyte system to explore the impact of particle size and charge upon mucus permeation, it has revealed in a robust manner the clear benefits for a particle possessing a neutral or near neutral surface. Beyond polyelectrolyte systems exploitation of polymer synthesis approaches such as living free radical chemistry should deliver stable, sizecontrolled nanoparticles possessing a uniform and balanced high density distribution of positive and negative surface charges, ultimately yielding particles with a net neutral surface potential that will provide an additional strategy to that of PEGylated systems when seeking to avoid interactions with the mucus barrier.

\section{LEGENDS}

Table 1. Particle physical characteristics and diffusion kinetics in mucus.

Figure 1: Relationships between particle diameter or zeta potential to particle diffusion kinetics in mucus. (1A) Plot of particle diameter of the polyelectrolyte (PAA:chitosan) NPs versus Effective Diffusion Coefficient $\langle$ Deff $>$; $(\underline{1 \mathrm{~B}})$ Plot of particle diameter of the polyelectrolyte NPs, the PEG-PLGA and the capsid virus AD5 versus Effective Diffusion 
Coefficient $<$ Deff $>$; $(\underline{1 \mathrm{C}})$ Plot of zeta potential of the polyelectrolyte NPs versus Effective Diffusion Coefficient <Deff>; (1D) Plot of zeta potential of the polyelectrolyte NPs, the PEG-PLGA and the capsid virus AD5 versus Effective Diffusion Coefficient $\langle$ Deff $\rangle$; $(1 \mathrm{E})$ Plot of zeta potential of the polyelectrolyte NPs versus \%ratio [Deff]/[D ${ }^{\circ}$, a measure of the efficiency of a particle's diffusion in mucus compared to its diffusion in water; (1F) Plot of zeta potential of the polyelectrolyte NPs, the PEG-PLGA and the capsid virus AD5 versus \%ratio $[$ Deff $] /\left[\mathrm{D}^{\circ}\right]$. Particle diameter is expressed in $\mathrm{nm}$, zeta potential is expressed in $\mathrm{mV}$ and $\langle$ Deff $\rangle$ is expressed in $\mathrm{cm}^{2} . \mathrm{s}^{-1} \times 10^{-9}$.

Figure 2: Three-dimensional response analysis showing the multi-variate relationship between particle diameter and zeta potential to polyelectrolyte particle diffusion kinetics in mucus. The polyelectrolyte particles were formed by self-assembly of various ratios of PAA and chitosan. Particle diameter is expressed in $\mathrm{nm}$, zeta potential is expressed in $\mathrm{mV}$ and the Effective Diffusion Coefficient $\langle$ Deff $\rangle$ is expressed in $\mathrm{cm}^{2} . \mathrm{s}^{-1} \times 10^{-9}$.

Figure 3: Heterogeneity of particle movement through mucus. For each particle type an effective diffusion coefficient (Deff) was calculated for each of 360 individual particles over a time interval of $1 \mathrm{sec}$. The data was ranked into percentiles from the $90^{\text {th }}$ through to $10^{\text {th }}$ percentile, where the $90^{\text {th }}$ percentile is the Deff value below which $90 \%$ of the Deff observations may be found. ( $\underline{3 \mathrm{~A}})$ data for the negatively charged [F1] polyelectrolyte formulation; $(\underline{3 \mathrm{~B}})$ data for the positively charged [F14] polyelectrolyte formulation; $(\underline{3 \mathrm{C}})$ data for the neutrally charged [F8] polyelectrolyte formulation; (3D) data for the PEG-PLGA particle. Figure presents data of from three separate experiments, i.e. $\geq 360$ individual particles examined for each particle type.

Supplementary Figure 1: Rheology of the mucus model. Strain sweep test of mucus in the \% strain range of 12 to 95 at which the viscoelastic plateau curve was obtained with the phase angle $\delta$ remaining constant. The storage modulus $\mathrm{G}^{\prime}$ values were higher the loss modulus values $\mathrm{G}^{\prime \prime}$ at all the \% strain tested values. The mucus was freshly isolated from pig intestinal ileum by gently scraping the intestinal surface to optimise the yield of not only the loose mucus layer but critically also a high content of the adherent mucus layer.

Supplementary Figure 2: AFM imaging of the mucus model. (S2A) AFM surface morphology of the mucus with large $(25 \times 25 \mu \mathrm{m})$, medium $(15 \times 15 \mu \mathrm{m})$ and small $(5 \times 5 \mu \mathrm{m})$ field images of the network of pores; $(\underline{\mathrm{S} 2 \mathrm{~B}})$ size distribution of over $98 \%$ of all visible pores within the mucus gel across ten randomly selected AFM fields each of $25 \times 25 \mu \mathrm{m}$.

Supplementary Figure 3: Illustrative profile of the Effective Diffusion Coefficients (Deff) for each of 20 randomly individual particles. The profiles show 20 particles from formulation [F8] across the time intervals of $1 \mathrm{sec}$. and $0.2 \mathrm{sec}$. Some particles display a rise in Deff across the $1 \mathrm{sec}$. interval indicative of diffusive transport; those particles that display such an increase represent the proportion of diffusive particles. The online source random.org was used to randomly select the particles. 
Supplementary Video 1: Video capture (x63 magnification oil immersion lens) across 300 frames (10 seconds) of the diffusive movements in intestinal porcine mucus of the $334 \mathrm{~nm}$ diameter polyelectrolyte neutral particles, i.e. [F8] formulation.

Supplementary Video 2: Video capture (x63 magnification oil immersion lens) across 300 frames (10 seconds) of the diffusive movements in intestinal porcine mucus of the $54 \mathrm{~nm}$ PEG-PLGA particles.

Supplementary Video 3: Video capture (x63 magnification oil immersion lens) across 300 frames (10 seconds) of the diffusive movements in intestinal porcine mucus of the $146 \mathrm{~nm}$ AD5 capsid virus.

\section{ACKNOWLEDGEMENTS}

Research supported by EC FP7 large-scale integrating project grant: Project number 280761 to the ALEXANDER consortium project "Mucus Permeating Nanoparticulate Drug Delivery Systems" in the topic area NMP-2011-1.2-2 "New targeted therapy using nanotechnology for transport of macromolecules across biological barriers"

\section{REFERENCES:}

[1] K. Park, I.C. Kwon, K. Park, Oral protein delivery: Current status and future prospect, Reactive and Functional Polymers. 71 (2011) 280-287. doi:10.1016/j.reactfunctpolym.2010.10.002.

[2] R.A. Cone, Barrier properties of mucus, Adv Drug Deliver Rev. 61 (2009) 75-85. doi:10.1016/j.addr.2008.09.008.

[3] K. Khanvilkar, M.D. Donovan, D.R. Flanagan, Drug transfer through mucus, Adv Drug Deliver Rev. 48 (2001) 173-193.

[4] M.M. Patel, J.D. Smart, T.G. Nevell, R.J. Ewen, P.J. Eaton, J. Tsibouklis, Mucin/poly(acrylic acid) interactions: a spectroscopic investigation of mucoadhesion, Biomacromolecules. 4 (2003) 1184-1190. doi:10.1021/bm034028p.

[5] C.A. Silva, T.M. Nobre, F.J. Pavinatto, O.N. Oliveira, Interaction of chitosan and mucin in a biomembrane model environment, J Colloid Interface Sci. 376 (2012) 289-295. doi:10.1016/j.jcis.2012.03.027.

[6] S.K. Lai, D.E. O'Hanlon, S. Harrold, S.T. Man, Y.-Y. Wang, R. Cone, et al., Rapid transport of large polymeric nanoparticles in fresh undiluted human mucus, Proc. Natl. Acad. Sci. U.S.a. 104 (2007) 1482-1487. doi:10.1073/pnas.0608611104.

[7] J.S. Suk, S.K. Lai, N.J. Boylan, M.R. Dawson, M.P. Boyle, J. Hanes, Rapid transport of muco-inert nanoparticles in cystic fibrosis sputum treated with $\mathrm{N}$-acetyl cysteine, Nanomedicine. 6 (2011) 365-375. doi:10.1016/j.

[8] S.S. Olmsted, J.L. Padgett, A.I. Yudin, K.J. Whaley, T.R. Moench, R.A. Cone, Diffusion of macromolecules and virus-like particles in human cervical mucus, Biophysj. 81 (2001) 1930-1937. doi:10.1016/S0006-3495(01)75844-4.

[9] L.J. Saif, E.H. Bohl, K.W. Theil, R.F. Cross, J.A. House, Particles Associated with 
Diarrhea in Young Pigst, 12 (1980) 105-111.

[10] F. Laffleur, F. Hintzen, G. Shahnaz, D. Rahmat, K. Leithner, A. Bernkop-Schnürch, Development and in vitro evaluation of slippery nanoparticles for enhanced diffusion through native mucus, Nanomedicine. 9 (2014) 387-396. doi:10.2217/nnm.13.26.

[11] Y. Cu, W.M. Saltzman, Drug delivery: Stealth particles give mucus the slip, Nature Materials. 8 (2009) 11-13. doi:10.1038/nmat2347.

[12] A.W. Larhed, P. Artursson, E. Björk, The influence of intestinal mucus components on the diffusion of drugs, Pharm Res. 15 (1998) 66-71.

[13] Y. Hu, X. Jiang, Y. Ding, H. Ge, Y. Yuan, C. Yang, Synthesis and characterization of chitosan-poly(acrylic acid) nanoparticles, Biomaterials. 23 (2002) 3193-3201.

[14] C.H. Bamford, H. Tompa, The theory of coacervation, Trans. Faraday Soc. 46 (1950) 310-316.

[15] C. Taylor, A. Allen, P.W. Dettmar, J.P. Pearson, Two rheologically different gastric mucus secretions with different putative functions, Biochim. Biophys. Acta. 1674 (2004) 131-138. doi:10.1016/j.bbagen.2004.06.007.

[16] J. Hanes, S.K. Lai, Compositions and methods for enhancing transport through mucus Patent PCT/US2007/019522, 2010.

[17] J. Suh, M. Dawson, J. Hanes, Real-time multiple-particle tracking: applications to drug and gene delivery, Adv Drug Deliver Rev. 57 (2005) 63-78. doi:10.1016/j.addr.2004.06.001.

[18] A. Macierzanka, A.R. Mackie, B.H. Bajka, N.M. Rigby, F.C.O. Nau, D. Dupont, Transport of particles in intestinal mucus under simulated infant and adult physiological conditions: impact of mucus structure and extracellular DNA, Plos One. 9 (2014) e95274. doi:10.1371/journal.pone.0095274.

[19] J. Philibert, One and a half century of diffusion: Fick, Einstein, before and beyond, Diffusion Fundamentals. 4 (2005) 1-19.

[20] C. Taylor, A. Allen, P.W. Dettmar, J.P. Pearson, The gel matrix of gastric mucus is maintained by a complex interplay of transient and nontransient associations, Biomacromolecules. 4 (2003) 922-927. doi:10.1021/bm025767t.

[21] R. Brunelli, M. Papi, G. Arcovito, A. Bompiani, M. Castagnola, T. Parasassi, et al., Globular structure of human ovulatory cervical mucus, Faseb J. 21 (2007) 38723876. doi:10.1096/fj.07-8189com.

[22] M. Dawson, Enhanced Viscoelasticity of Human Cystic Fibrotic Sputum Correlates with Increasing Microheterogeneity in Particle Transport, Journal of Biological Chemistry. 278 (2003) 50393-50401. doi:10.1074/jbc.M309026200.

[23] H. Shen, Y. Hu, W.M. Saltzman, DNA diffusion in mucus: effect of size, topology of DNAs, and transfection reagents, Biophysj. 91 (2006) 639-644. doi:10.1529/biophysj.105.077404.

[24] T.A.S. Etrych, L. Leclercq, M. Boustta, M. Vert, Polyelectrolyte complex formation and stability when mixing polyanions and polycations in salted media: a model study related to the case of body fluids, Eur J Pharm Sci. 25 (2005) 281-288. doi:10.1016/j.ejps.2005.03.005.

[25] M.P. Deacon, S.S. Davis, R.J. White, H. Nordman, I. Carlstedt, N. Errington, et al., Are chitosan-mucin interactions specific to different regions of the stomach? Velocity ultracentrifugation offers a clue, Carbohydrate Polymers. 38 (1999) 235238. doi:10.1016/S0144-8617(98)00097-6.

[26] I.A. Sogias, A.C. Williams, V.V. Khutoryanskiy, Why is chitosan mucoadhesive? Biomacromolecules. 9 (2008) 1837-1842. doi:10.1021/bm800276d.

[27] S.A. Mortazavi, An in vitro assessment of mucus/mucoadhesive interactions, Int J Pharm. 124 (1995) 173-182. doi:10.1016/0378-5173(95)00073-R. 
[28] S.K. Lai, Y.-Y. Wang, D. Wirtz, J. Hanes, Micro- and macrorheology of mucus, Adv Drug Deliver Rev. 61 (2009) 86-100. doi:10.1016/j.addr.2008.09.012.

[29] Y.-Y. Wang, S.K. Lai, J.S. Suk, A. Pace, R. Cone, J. Hanes, Addressing the PEG Mucoadhesivity Paradox to Engineer Nanoparticles that "Slip" through the Human Mucus Barrier, Angew. Chem. Int. Ed. 47 (2008) 9726-9729. doi:10.1002/anie.200803526.

[30] B.C. Tang, M. Dawson, S.K. Lai, Y.-Y. Wang, J.S. Suk, M. Yang, et al., Biodegradable polymer nanoparticles that rapidly penetrate the human mucus barrier, Proc. Natl. Acad. Sci. U.S.a. 106 (2009) 19268-19273. doi:10.1073/pnas.0905998106.

[31] J.S. Suk, S.K. Lai, Y.-Y. Wang, L.M. Ensign, P.L. Zeitlin, M.P. Boyle, et al., The penetration of fresh undiluted sputum expectorated by cystic fibrosis patients by nonadhesive polymer nanoparticles, Biomaterials. 30 (2009) 2591-2597. doi:10.1016/j.biomaterials.2008.12.076.

[32] A. Šiber, A.L. Božič, R. Podgornik, Energies and pressures in viruses: contribution of nonspecific electrostatic interactions, Phys Chem Chem Phys. 14 (2012) 37463765. doi:10.1039/c1cp22756d.

[33] A. Lošdorfer Božič, A. Šiber, R. Podgornik, How simple can a model of an empty viral capsid be? Charge distributions in viral capsids, J Biol Phys. 38 (2012) 657671. doi:10.1007/s10867-012-9278-4.

[34] B. Michen, T. Graule, Isoelectric points of viruses, J. Appl. Microbiol. 109 (2010) 388-397. doi:10.1111/j.1365-2672.2010.04663.x.

[35] J.R. Stonebraker, D. Wagner, R.W. Lefensty, K. Burns, S.J. Gendler, J.M. Bergelson, et al., Glycocalyx restricts adenoviral vector access to apical receptors expressed on respiratory epithelium in vitro and in vivo: role for tethered mucins as barriers to lumenal infection, J. Virol. 78 (2004) 13755-13768. doi:10.1128/JVI.78.24.1375513768.2004.

[36] C. Kitson, B. Angel, D. Judd, S. Rothery, N.J. Severs, A. Dewar, et al., The extraand intracellular barriers to lipid and adenovirus-mediated pulmonary gene transfer in native sheep airway epithelium, Gene Ther. 6 (1999) 534-546. doi:10.1038/sj.gt.3300840.

[37] L.D. Li, T. Crouzier, A. Sarkar, L. Dunphy, J. Han, K. Ribbeck, Spatial configuration and composition of charge modulates transport into a mucin hydrogel barrier, Biophys. J. 105 (2013) 1357-1365. doi:10.1016/j.bpj.2013.07.050. 
Table 1 Particle physical characteristics and diffusion kinetics in mucus.

\begin{tabular}{|c|c|c|c|c|c|c|c|c|}
\hline Nature of NPs & $\begin{array}{c}\text { PAA:Chitosan } \\
\text { Mass ratio }\end{array}$ & Code & $\begin{array}{l}\text { Zeta Potential } \\
(\mathrm{mV}) \\
\text { Mean ( } \pm \text { s.d. })\end{array}$ & $\begin{array}{c}\text { Particle } \\
\text { Diameter } \\
(\mathrm{nm}) \\
\text { Mean (PDI) }\end{array}$ & $\begin{array}{c}\mathrm{D}^{\circ} \text { (water) } \\
\mathrm{cm}^{2} \cdot \mathrm{S}^{-1} \times 10^{-9}\end{array}$ & $\begin{array}{l}<\text { Deff }>\text { (mucus) } \\
\mathrm{cm}^{2} \cdot \mathrm{S}^{-1} \times 10^{-9} \\
\text { Mean }( \pm \text { s.e.m) }\end{array}$ & $\begin{array}{l}\% \text { Ratio } \\
<\text { Deff }>/ \\
\mathrm{D}^{\circ}\end{array}$ & $\begin{array}{c}\% \\
\text { Diffusive } \\
\text { particles }\end{array}$ \\
\hline \multirow{6}{*}{$\begin{array}{l}\text { Negatively } \\
\text { charged } \\
\text { Polyelectrolyte }\end{array}$} & $5: 1$ & F1 & $-29.1( \pm 3.6)$ & $104(0.14)$ & 43.21 & $0.00163( \pm 0.00031)$ & 0.0038 & 1 \\
\hline & $4: 1$ & $\mathrm{~F} 2$ & $-30.6( \pm 4.4)$ & $149(0.10)$ & 30.16 & $0.00366( \pm 0.00067)$ & 0.0121 & 2 \\
\hline & $3: 1$ & F3 & $-25.0( \pm 4.0)$ & $204(0.21)$ & 22.03 & $0.01781( \pm 0.00378)$ & 0.0809 & 6 \\
\hline & $2: 1$ & $\mathrm{~F} 4$ & $-18.9( \pm 1.3)$ & $225(0.13)$ & 19.97 & $0.01574( \pm 0.00329)$ & 0.0788 & 4 \\
\hline & $1: 1$ & F5 & $-15.4( \pm 0.8)$ & $357(0.21)$ & 12.59 & $0.02286( \pm 0.00534)$ & 0.1816 & 5 \\
\hline & $1: 1 *$ & F6 & $-15.2( \pm 1.2)$ & $365(0.21)$ & 12.31 & $0.01749( \pm 0.00365)$ & 0.1421 & - \\
\hline \multirow{2}{*}{$\begin{array}{l}\text { Neutral } \\
\text { Polyelectrolyte }\end{array}$} & $1: 2.2$ & F7 & $-0.5( \pm 1.9)$ & $1244(0.32)$ & 3.61 & $0.00239( \pm 0.00064)$ & 0.0661 & 1 \\
\hline & $1: 2.2 *$ & $\mathrm{~F} 8$ & $+1.1( \pm 2.4)$ & $334(0.19)$ & 13.46 & $0.02887( \pm 0.00501)$ & 0.2146 & 13 \\
\hline \multirow{6}{*}{$\begin{array}{l}\text { Positively } \\
\text { charged } \\
\text { Polyelectrolyte }\end{array}$} & $1: 3$ & F9 & $+6.0( \pm 1.1)$ & $144(0.18)$ & 31.21 & $0.03182( \pm 0.00650)$ & 0.1019 & 9 \\
\hline & $1: 4$ & F10 & $+14.3( \pm 0.3)$ & $104(0.09)$ & 43.21 & $0.00849( \pm 0.00213)$ & 0.0196 & 2 \\
\hline & $1: 5$ & F11 & $+19.2( \pm 0.5)$ & $180(0.17)$ & 24.97 & $0.00104( \pm 0.00018)$ & 0.0042 & 1 \\
\hline & $1: 6$ & F12 & $+19.5( \pm 0.9)$ & $293(0.17)$ & 15.34 & $0.00084( \pm 0.00014)$ & 0.0055 & 1 \\
\hline & $1: 8$ & F13 & $+19.2( \pm 0.6)$ & $359(0.11)$ & 12.52 & $0.00031( \pm 0.00009)$ & 0.0024 & 1 \\
\hline & $1: 8^{*}$ & F14 & $+19.5( \pm 2.3)$ & $373(0.23)$ & 11.92 & $0.00032( \pm 0.00006)$ & 0.0030 & - \\
\hline Lipophilic & PLGA & - & $-29.2( \pm 2.1)$ & $161(0.03)$ & 27.91 & $0.00013( \pm 0.00002)$ & 0.0005 & 1 \\
\hline Hydrophillic & PEG-PLGA & - & $-8.3( \pm 1.2)$ & $54(0.03)$ & 83.22 & $0.46889( \pm 0.09343)$ & 0.5634 & 32 \\
\hline Capsid Virus & AD5 & - & $-0.5( \pm 2.3)$ & $146(0.18)$ & 30.78 & $0.04391( \pm 0.00891)$ & 0.1426 & 19 \\
\hline
\end{tabular}

* indicates NPs sonicated after fabrication prior to diffusion measurement. 

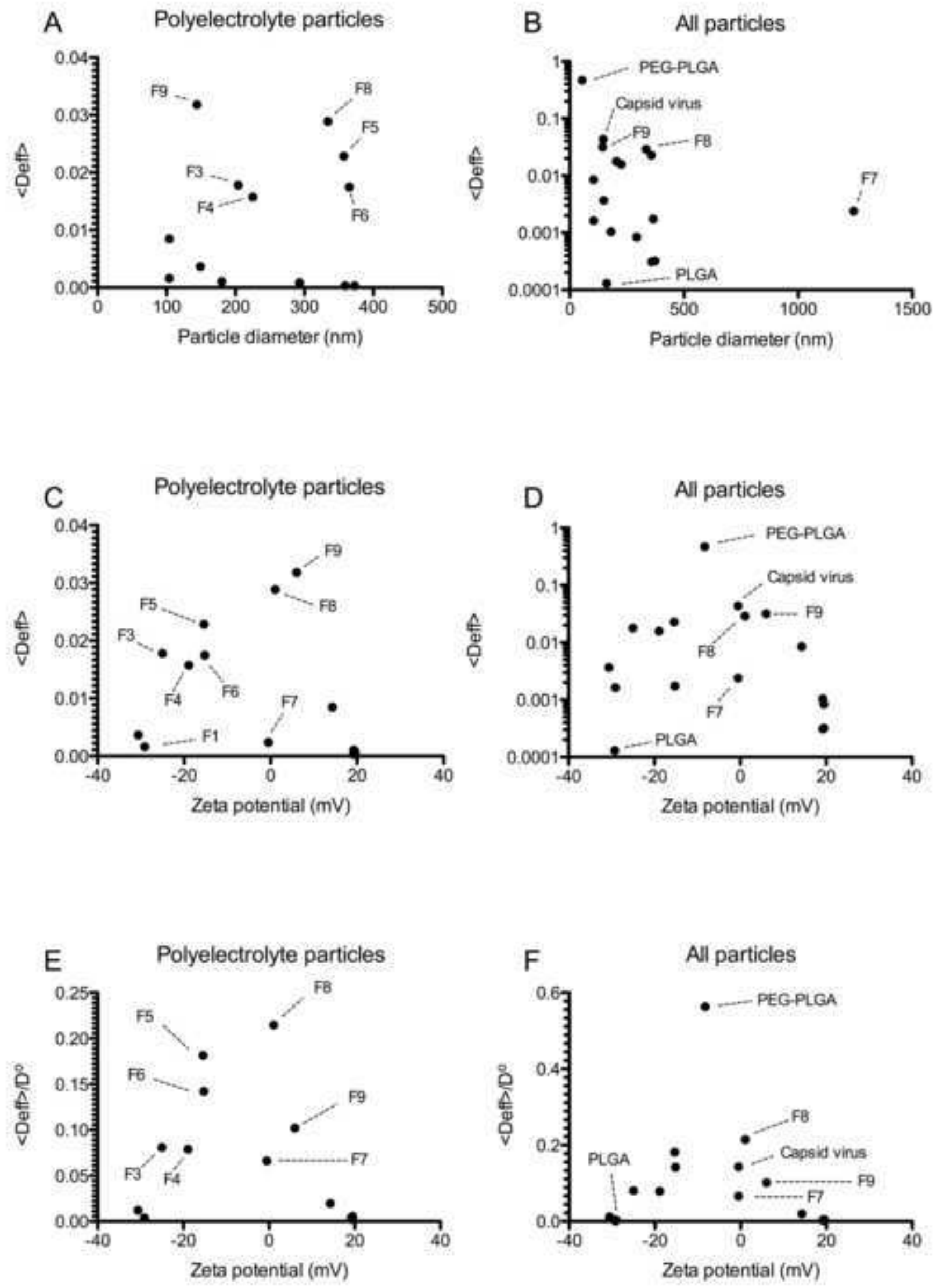
Figure 2

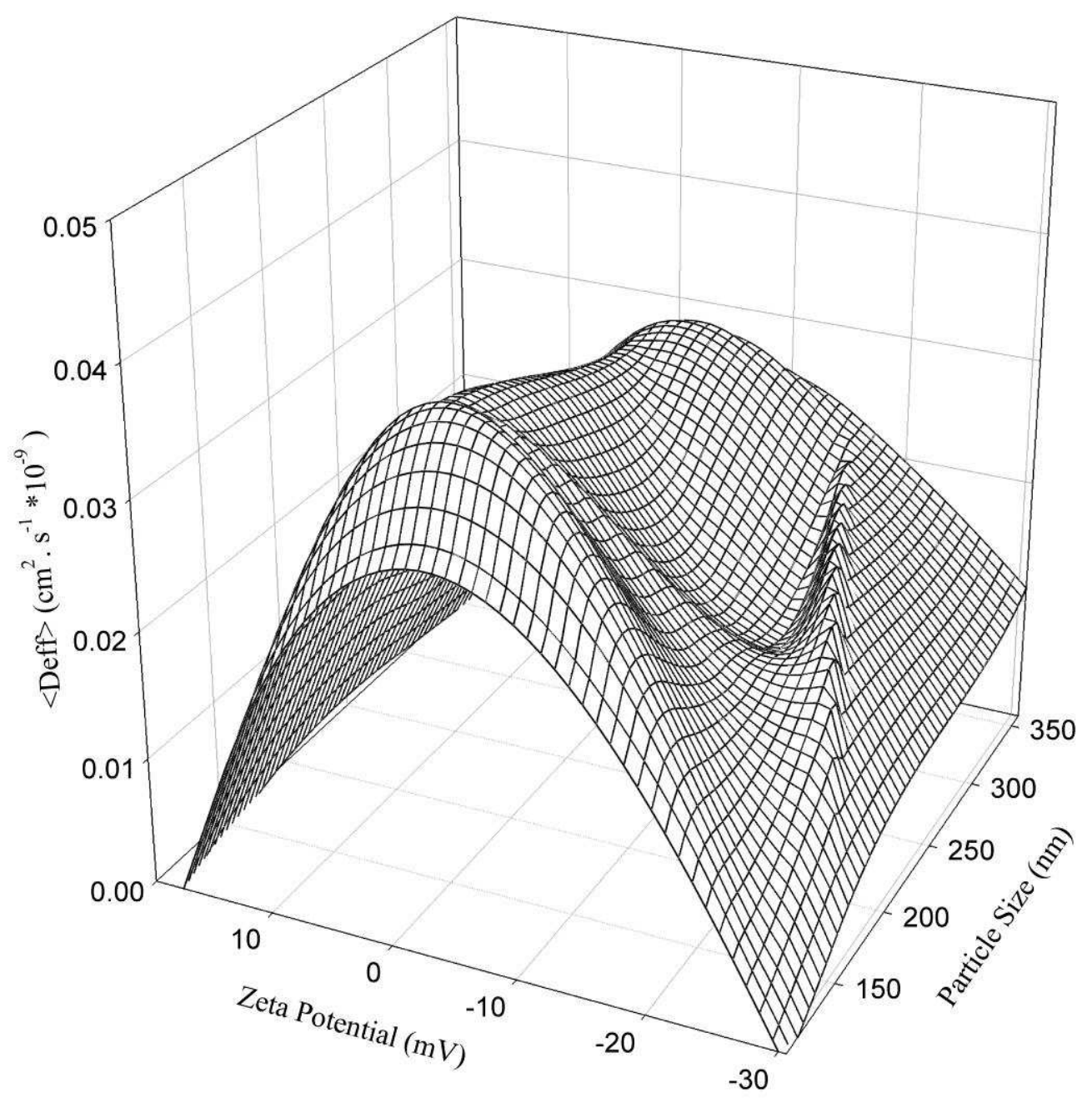


Click here to download high resolution image
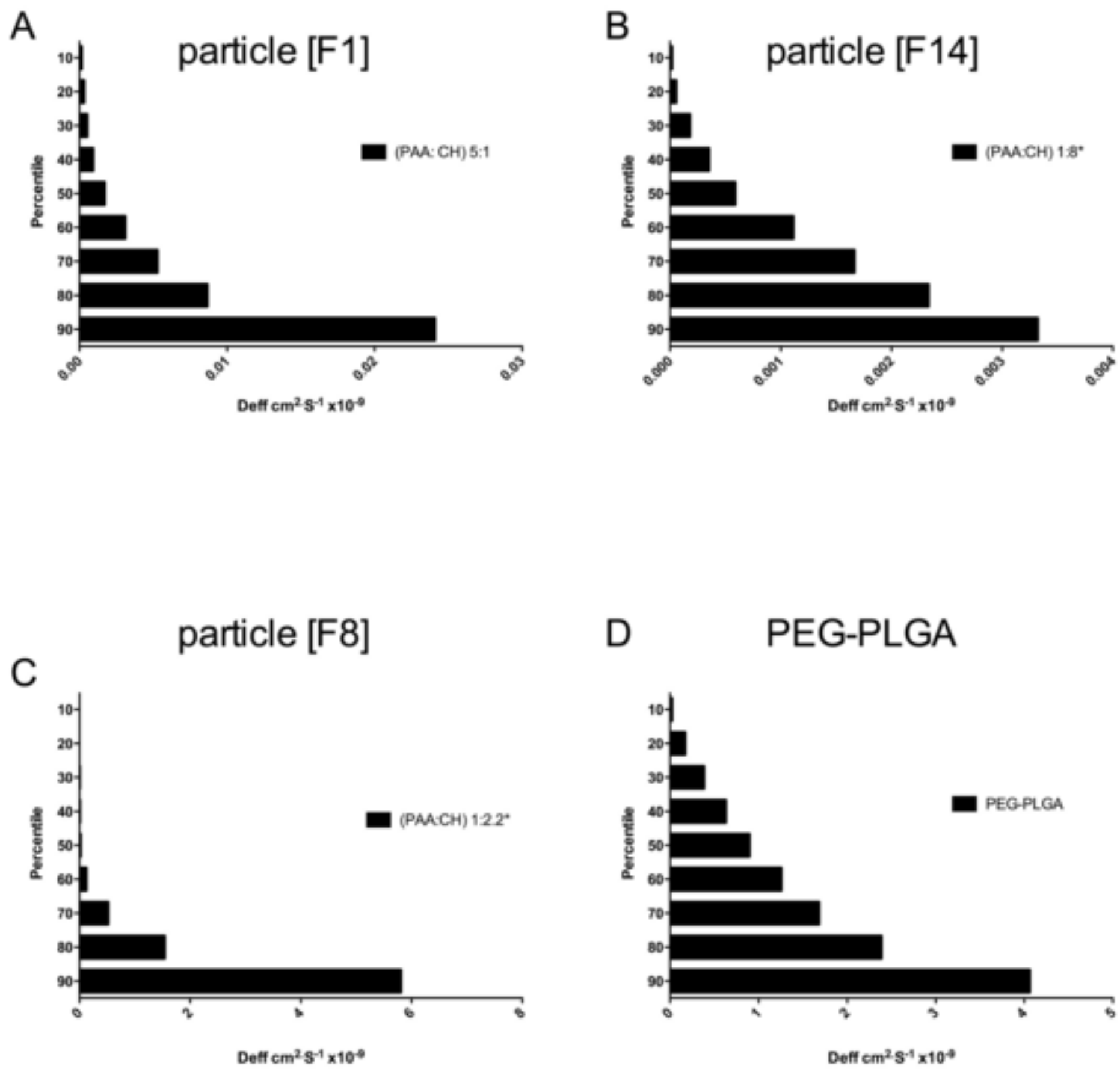

D
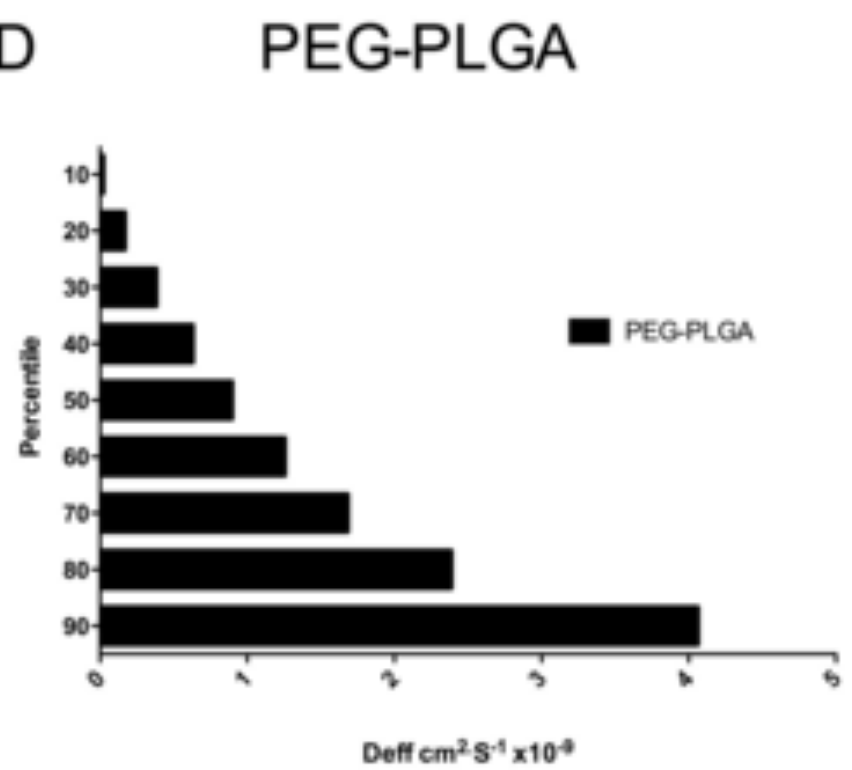

Supplementary Figure 1
Click here to download Supplementary material: Supplementary Figure 1.tiff (1)

ff

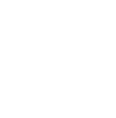

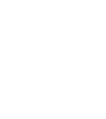

Figure 1. 
Supplementary Figure 2
Click here to download Supplementary material: Supplementary Figure 2.docx

Supplementary Figure 2
Click here to download Supplementary material: Supplementary Figure 2.docx

\author{
Ch here to download Supplementary material: Supplementary Figure 2.docx
}

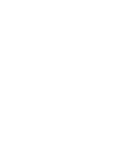

Figure 2.docx

政

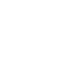

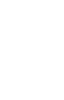

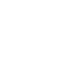

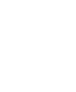

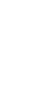
. 更 更 更 更 更 更 更 更 更

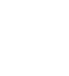
更 更 更 更 更 更 更 更

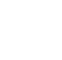



Supplementary Figure 3
Click here to download S

Click here to download Supplementary material: Supplementary Figure 3.tiff 

Supplementary video 1
Click here to download Supplementary material: Supplementary video 1.mov

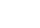


Supplementary video 2
Click here to download Supplementary material: Supplementary video 2.mov

\begin{abstract}
click here to download Supplementary material Supplementary video 2 mov
\end{abstract} (1)

Supplementary material: Supplementary video 2 mov

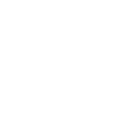
(1) (1) (1) (1) (1)

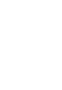
(1) (1)

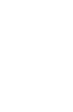
(1) (1) (1) . . . . . . . . . . . . 
Supplementary video 3
Click here to download Supplementary material: Supplementary video 3.mov

\begin{abstract}
click here to download Supplementary material: Supplementary video $3 . \mathrm{mov}$
\end{abstract} (1)

Supplementary material Supplementary video 3 mov

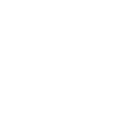

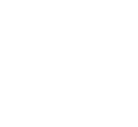

(1)

$\sqrt{2}$
(1) $\sqrt{3}$

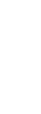
(1) (1) (1)

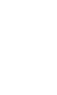
. . . . . . . . . . . . . . . . 


\section{ABBREVIATIONS}

\begin{tabular}{|c|c|c|c|}
\hline AD5 & Adenovirus & $\mathrm{mV}$ & Millivolt \\
\hline $\mathrm{AFM}$ & Atomic force microscopy & $\mathrm{nm}$ & Nanometer \\
\hline $\mathrm{D}^{\circ}$ & Absolute diffusion & $\mathrm{NP}(\mathrm{s})$ & Nanoparticle(s) \\
\hline Deff & Effective diffusion coefficient & PAA & Polyacrylic acid \\
\hline$<$ Deff $>$ & $\begin{array}{l}\text { Ensemble effective diffusion } \\
\text { coefficient }\end{array}$ & PAM & Polyallylamine \\
\hline DF & Diffusivity factor & PBS & Phosphate buffer saline \\
\hline $\mathrm{G}^{\prime}$ & Storage modulus & PDI & Poly Dispersity Index \\
\hline$G^{\prime \prime}$ & Loss modulus & PEC NPs & Polyelectrolyte Nanoparticles \\
\hline GIT & Gastrointestinal tract & PEG-NP & PEGylated nanoparticles \\
\hline IEP & Isoelectric point & PLGA & Poly(lactic-co-glycolic acid) \\
\hline $\mathrm{K}$ & Boltzmann constant & $\mathrm{r}$ & Radius of Nanoparticles \\
\hline $\mathrm{KHz}$ & Kilo hertz & SD & Square Displacement \\
\hline LR & Lumogen Red 305 & s.d. & Standard deviation \\
\hline MPT & Multiple particle tracking & $\mathrm{Sec}$ & Second \\
\hline MSD & Mean square displacement & s.e.m & Standard error of mean \\
\hline$<\mathrm{MSD}>$ & $\begin{array}{l}\text { Ensemble mean square } \\
\text { displacement }\end{array}$ & $\mathrm{t}$ & Time interval \\
\hline$\eta$ & Water viscosity & $\mathrm{T}$ & Absolute temperature \\
\hline$\mu \mathrm{m}$ & Micrometre & $\delta$ & Phase angle \\
\hline$+\mathrm{ve}$ & Positive charge & -ve & Negative charge \\
\hline
\end{tabular}

